\title{
DISTRIBUIÇÃO ESPACIAL DE CRIADOUROS DE AEDES AEGYPTI EM JAGUARUANA - CE - BRASIL E SUAS CORRELAÇÕES COM INDICADORES SOCIODEMOGRÁFICOS
}

\section{SPACE DISTRIBUTION OF AEDES AEGYPTI BREEDING SITES IN JAGUARUANA - CE - BRAZIL AND ITS CORRELATIONS WITH SOCIODEMOGRAPHIC INDICATORS}

\author{
Carlos Vangerre de Almeida Maia \\ Mestre em Desenvolvimento e Meio Ambiente - Universidade Federal do Piauí (UFPI) \\ Consórcio de Gestão Integrada de Resíduos Sólidos - Vale do Jaguaribe (CGIRS-VJ) \\ cvamaia@yahoo.com.br \\ Gledson Santos de Lima \\ Geógrafo. Especialista em Geoprocessamento - Universidade Estadual do Ceará (UECE) \\ Instituto de Estudos, Pesquisas e Projetos (IEPRO/UECE) \\ gledsonsli@yahoo.com.br \\ Alisson Daniel Silva Rocha \\ Acadêmico de Tecnologia em Saneamento Ambiental \\ Instituto Federal de Educação, Ciência e Tecnologia do Ceará (IFCE) \\ alisson.daniel3000@gmail.com \\ Estelita Lara de Oliveira \\ Acadêmica de Psicologia - Faculdade Vale do Jaguaribe (FVJ) \\ estelitalara.jp2000@gmail.com \\ Márcia Cristina Freitas da Silva \\ Acadêmica de Biomedicina - Faculdade de Enfermagem Nova Esperança (FACENE) \\ marciacrisf@gmail.com \\ Hihara Henrique da Silva \\ Acadêmica de Licenciatura Plena em Letras - Universidade Estadual do Ceará (UECE) \\ hiharaenrique16@gmail.com \\ Gabriel Douglas Santiago Barros \\ Acadêmico de Licenciatura Plena em Geografia - Universidade Estadual do Ceará (UECE) \\ gabriel.d.uece@gmail.com
}

\begin{abstract}
RESUMO
O Aedes aegypti é originário das selvas africanas, principal responsável pela disseminação de arboviroses (Dengue, Febre amarela, Zika e Chikungunya), capaz de se reproduzir em ambientes naturais ou depósitos artificiais. O controle das arboviroses passa pela capacidade de eliminar os Depósitos Positivos (DPs) para os culicídeos. O objetivo desse trabalho é mapear as áreas de risco para a ocorrência de DPs em três bairros contíguos em Jaguaruana-CE, referente aos anos 2014 e 2015, mediante análises geoestatísticas, bem como aferir possíveis correlações entre variáveis sociodemográficas, constantes nos 14 setores censitários que se sobrepõem aos bairros e a ocorrência dos DPs, através da Correlação de Spearman, adotando o Intervalo de Confiança menor ou igual a $95 \%$. Os resultados comprovam a existência de uma possível área de risco, bem como, correlação entre a DPs e densidade populacional $(\rho=0,58 ; p=0,03)$ e com a coleta de resíduos sólidos $(\rho=-0,53 ; p=0,05)$, coadunando para o entendimento de que o mosquito, por meio da oferta de seres humanos para praticar a hematofagia e existência de depósitos para sua ovipostura, não precisa se deslocar muito. Ou seja, as atuais condições são favoráveis para sua perpetuação na região estudada, cabendo ao poder público local se munir de estratégias para o combate ao culicídeo.
\end{abstract}

Palavras-chave: Arboviroses. Depósitos Positivos. Geoestatística.

Recebido em: 07/01/2019

Aceito para publicação em: 06/05/2019 


\begin{abstract}
The Aedes aegypti originates from the African jungles, the main one responsible for the dissemination of arboviruses (Dengue, Zika and Chikungunya), able to reproduce in natural environments or artificial deposits. The control of arboviruses involves the ability to eliminate Positive Deposits (PDs) for culicidae. The aim of this work is to map the risk areas for the occurrence of PDs (Positive Deposits) in three contiguous neighborhoods, in Jaguaruana$\mathrm{CE}$, for the years 2014 and 2015, through geostatistical analysis, as well as to measure possible correlations between constant sociodemographic variables in 14 census ares that overlap the neighborhoods and the occurrence of PDs, through the Spearman Correlation, adopting the confidence interval less than or equal to $95 \%$. The results confirm the existence of a possible risk area, and a correlation between PDs and the population density $(\rho=0.58$, $p=0.03)$ and solid waste collection $(\rho=0.53 ; p=0.05)$, combining for the understanding that the mosquito does not have to move much due to the supply of human beings to practice hematophagy and the existence of deposits for its oviposition. This means that the current conditions are favorable for its perpetuation in the region under study, and it is up to the local public power to provide strategies to combat Culicidae.
\end{abstract}

Key words: Arboviruses. Positive Deposits. Geostatistics.

\title{
INTRODUÇÃO
}

O Aedes aegypti pertence a família Culicidae, apresenta hábitos antropofílicos, cujas fêmeas necessitam realizar hematofagia para a sua ovopostura, sendo o principal responsável pela disseminação de arboviroses, como Dengue, Zika, Chikungunya e Febre Amarela.

De origem africana, sua introdução se remonta ao período Colonial, possivelmente quando do tráfico de escravos, sendo que os primeiros casos de Dengue foram registrados em 1865, Já os casos de Chykungunia e Zika respectivamente, tiveram seus primeiros casos autóctones detectados em 2014 e 2015, todos na Região Nordeste (TONIAZZO et al., 2016; ZARA et al., 2016; CAVALCANTI et al., 2018).

Embora selvagem, por condições ambientais, sanitárias, econômicas e sociais, o culicídeo também se reproduz em ambientes urbanos, onde reside a maior parte da população brasileira, espaços por vezes marcados pela ausência de planejamento, seletividade quanto ao acesso à serviços de saneamento básico, habitação, dentre outras infraestruturas e equipamentos sociais.

Essas deficiências estruturais favorecem os surgimentos de criadouros de Ae. aegypti, cuja existência desses depósitos em regiões de maior vulnerabilidade social, onde residem grupos populacionais com menor capacidade de resposta, se configura como um grave problema socioeconômico, epidemiológico e de promoção de saúde (CARVALHO, et al., 2017).

O combate às arboviroses passa, pelo controle dos criadouros, onde, em maior quantidade, são encontrados no intra e no peridomicílio, podendo ser controlados sob formas mecânica, química, física, biológica, cultural e legal (TONIAZZO et al., 2016; SOBRAL, SOBRAL, 2017; CARVALHO et al., 2017).

O monitoramento de infestação do Ae. aegypti, no Brasil, ocorre por meio de visitas diárias dos Agentes Comunitários de Endemias (ACE) Levantamento de Índice Rápido para Aedes aegypti (LIRAa), que veio a se tornar obrigatório apenas em 2016, mediante Portaria $n^{\circ} 3129 / 2016$ do Ministério da Saúde, consistindo em uma amostragem larvária bimestral do vetor em um município, através de três índices:

Índice de Infestação Predial - percentual de imóveis com presença de criadouros positivos para larvas de Ae. aegypti; Índice de Breteau - no de criadouros positivos para larvas do culicídeo em 100 imóveis; Índice de Recipiente - Distribuição percentual de cada tipo de recipiente, em relação ao total de criadouros encontrados; 
Porém, de acordo com Marteis et al. (2013), essas técnicas podem ser ineficientes, para estimar a densidade e produtividade dos criadouros, havendo episódios de surtos arboviróticos em regiões onde os índices calculados não demonstravam risco².

Logo, se faz necessário que outras técnicas possam também fazer parte do plano estratégico para o controle dos criadouros, sobretudo, buscando analisar e fazer interlocuções com os espaços onde eles se encontram, possibilitando a identificação de risco e aventar suas causas, já que a distribuição de episódios de arboviroses podem não se distribuir de maneira equânime, como também pode haver heterogeneidade espacial entre ocorrências de casos e criadouros (JOHANSEN et al., 2014; TONIAZZO, 2016).

Portanto, se faz fulcral conhecer o comportamento espaço-temporal larvário e os tipos de criadouros onde eles se desenvolvem, como primeiro passo para o enfrentamento ao mosquito, onde, para isso a utilização de técnicas de análises espaciais pode favorecer a vigilância e controle do $A$. aegypti.

Tanto que, em 2016, no Relatório da Reunião Internacional para Implementação de Alternativas para o Controle do Ae. aegypti no Brasil, recomendou-se que algumas tecnologias sejam incluídas nas diretrizes do Programa Nacional de Controle da Dengue, dentre as quais o mapeamento de risco, que consiste em avaliar e identificar áreas de risco aumentado para transmissão de arboviroses, utilizando estatísticas espaciais ${ }^{3}$ locais, relacionado os dados espaciais com dados da vigilância entomológica, da vigilância epidemiológica e das redes laboratoriais e de saneamento, permitindo que as ações específicas de controle vetorial possam ser direcionadas a áreas prioritárias. (BRASIL, 2016).

Logo, o objetivo deste trabalho é analisar e caracterizar a distribuição espacial de criadouros de $A$. aegypti em três bairros contíguos em Jaguaruana-CE, entre os anos 2014 e 2015, através de técnicas de estatística espacial, além de compreender padrões e possíveis relações com variáveis sociodemográficas levantadas pelo Censo Demográfico do Brasil de 2010.

\section{MATERIAIS E MÉTODOS}

O estudo é do tipo descritivo e ecológico, realizado no município de Jaguaruana - CE, Brasil, situado na região do Litoral Leste cearense, distante, aproximadamente $210 \mathrm{~km}$ ao sudeste de Fortaleza, onde foram escolhidos três bairros contíguos e que fossem de fácil acesso: Centro, Juazeiro e Lagoa.

Esses bairros, conjuntamente, de acordo com o Instituto Brasileiro de Geografia e Estatística (IBGE). (2010), continham 9446 habitantes, representando cerca de $29,3 \%$ da população total do município, bem como 49,36\% da população urbana de Jaguaruana - CE, além de terem 14 setores censitários contidos nesse território, conforme Figura 1.

Junto à Secretaria Municipal de Saúde, solicitou-se os endereços de onde foram identificados DPs, que, mediante o aplicativo gratuito para smartphone C7 GPS Dados, criado pelo Laboratório de Geomática da Universidade Federal de Santa Maria, foram obtidas as coordenadas em projeção cartográfica Universal Transversa de Mercator (UTM) dos respectivos endereços, oriundos tanto do Levantamento Rápido, realizado pelos ACEs, como dos endereços denunciados pela população, onde foi possível criar um arquivo de pontos georreferenciados.

Para a delimitação dos bairros onde se enquadravam os endereços, foram utilizados os croquis das áreas de atuação dos Agentes de Controle de Endemias (ACE). Já os setores censitários a serem utilizados nessa pesquisa, foram obtidos mediante a base cartográfica proveniente do portal do IBGE.

\footnotetext{
${ }^{2}$ Sobre as críticas que os atuais índices larvários recebem, Silva e Limongi (2018), indicam que armadilhas, em especial as ovitrampas, são instrumentos sensíveis e confiáveis para identificar o potencial de transmissão do vetor, podendo refletir a prevalência e o potencial de transmissão de vetores nos territórios, gerar dados epidemiológicos para a vigilância em saúde, medidas de controle, além de mostrar a detecção ou não da espécie em determinada área.

${ }^{3}$ Entende-se que a estatística espacial é o ramo da estatística que nos permite modelar a ocorrência de fenômenos que expõem explicitamente uma dependência espacial, inserindo, por exemplo, fatores determinantes, a estrutura de distribuição espacial ou a identificação de padrões. (SANTOS e SOUZA, 2008).
} 
Distribuição espacial de criadouros de Aedes aegypti em Jaguaruana - CE - Brasil e suas correlações com indicadores sociodemográficos
Carlos Vangerre de Almeida Maia Gledson Santos de Lima

Alisson Daniel Silva Rocha

Estelita Lara de Oliveira

Márcia Cristina Freitas da Silva

Hihara Henrique da Silva

Gabriel Douglas Santiago Barros

Figura 1 - Mapa de localização de Jaguaruana, Ceará - Brasil.

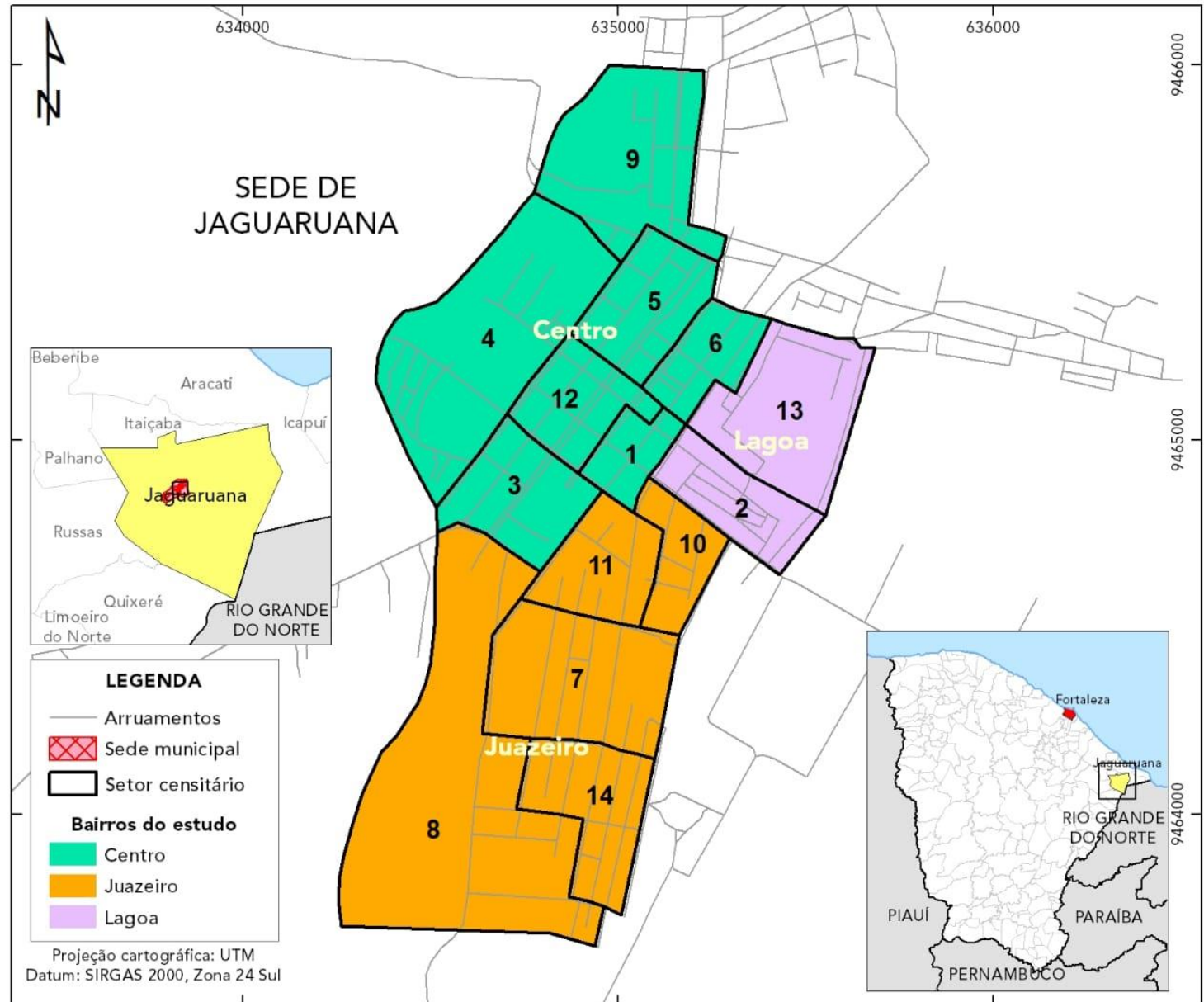

Fonte: própria.

Os dados de arruamentos da cidade foram adquiridos da base de mapas do projeto de mapeamento colaborativo OpenStreetMap, o qual disponibiliza abertamente os mapas e metadados da superfície terrestre, que foram armazenados em uma estrutura de banco de dados espaciais para análise em um programa de Sistema de Informação Geográfica (SIG). Para esse estudo, foi utilizada uma versão de teste do software ArcGIS, desenvolvido pela empresa Environmental Sytems Research Institute (ESRI).

Em seguida, realizou-se análise descritiva referente às características dos tipos de depósitos, desencadeando no padrão espacial de suas ocorrências, identificando o centro médio e a elipse de desvio padrão ${ }^{4}$.

A posteriori, criou-se uma faixa de distância com raio de 200 metros para cada foco, possibilitando visualizar uma possível área de dispersão do mosquito, bem como, utilizando a estimativa Kerne/ ${ }^{5}$, buscou-se identificar possíveis áreas de risco.

\footnotetext{
${ }^{4}$ De acordo com Ebdon (1985 apud TARTARUGA, 2009), a elipse de desvio padrão é derivada a partir da distribuição bivariada do desvio padrão das coordenadas nas direções $\mathrm{X}$ e $\mathrm{Y}$. Todavia, a elipse é calculada em duas etapas. Primeiro é calculada a orientação dos eixos da elipse de modo que a soma dos quadrados seja mínimo e se tenha o ângulo dessa orientação. E depois o eixo Y é rotacionados no sentido horário por esse ângulo e são calculados os desvios padrões utilizando os eixos $\mathrm{X}$ e $\mathrm{Y}$ girados e partir destes são calculados os comprimentos dos eixos.
} 
Distribuição espacial de criadouros de Aedes aegypti em Jaguaruana - CE - Brasil e suas correlações com indicadores sociodemográficos
Carlos Vangerre de Almeida Maia Gledson Santos de Lima

Alisson Daniel Silva Rocha Estelita Lara de Oliveira Márcia Cristina Freitas da Silva Hihara Henrique da Silva Gabriel Douglas Santiago Barros

Após a identificação das áreas de risco, foram selecionados/calculados indicadores socioeconômicos, sanitários e ambientais formulados a partir de Brasil (2011), incluindo condições de habitação, saneamento, renda, analfabetismo e escolaridade.

Mediante a Correlação de Spearman ${ }^{6}$, identificou-se quais das variáveis mantiveram correlação significativa com o número de DPs, onde, para esse estudo foi adotada significância menor ou igual a $95 \%$ ( $p \leq 0,05)$, ao longo dos 14 setores censitários que se sobrepõem ao território dos três bairros.

\section{RESULTADOS E DISCUSSÃO}

No Ceará, há casos de dengue notificados desde 1986, havendo, desde então, sete grandes epidemias nos anos de 1987, 1994, 2001, 2008, 2011, 2012 e 2015. Nesse último ano, também foi confirmada a transmissão autóctone dos vírus da chikungunya e da zika (CEARÁ, 2017).

Se o mosquito já era de grande relevância à saúde pública no âmbito nacional, no cenário estadual, então, é que suas relações ecobiossociais devem ser melhor estruturadas, vide que, mesmo sendo os primeiros registros autóctones de chikungunya e zika datado de 2015, em 2017, o Ceará liderou o ranking da incidência de arboviroses no Brasil (BRASIL, 2018).

Um ano antes, em 2016, Jaguaruana, dentro da sua regional de saúde, composta por cinco municípios, obtinha a segunda maior incidência de Chikungunya: e $65^{\circ}$ maior incidência cearense, num total de 184 municípios (CEARÁ, 2016).

De acordo com Brasil (2013), lavas e pupas do A. aegypti podem ser encontradas em vários depósitos, cuja classificação utilizada é: A1 - caixa d'água; A2 - depósitos de armazenamento de água ao nível do solo; B - pequenos depósitos móveis; C - depósitos fixos; D1 - pneus e outros materiais rodantes; D2 - lixo, recipientes de plástico, latas, sucatas, entulhos; E - depósitos naturais.

No período, foram identificados 27 endereços com DPs no bairro Juazeiro, 59 na Lagoa e 55 no Centro, totalizando 141 endereços. Porém, para essa pesquisa se tornaram válidos 24 endereços no Juazeiro $(88,8 \%), 53(89,8 \%)$ na Lagoa, e 47 no Centro $(85,4 \%)$, pois alguns endereços não foram encontrados ou por inconsistência no registro, totalizando cerca de $87,9 \%$ do universo, apresentados na Tabela 1.

Tabela 1 - Depósitos positivos para Aedes aegypti

\begin{tabular}{ccccccccc}
\hline \multirow{2}{*}{ Bairros } & \multicolumn{1}{c}{ Tipos de Deposito } \\
\cline { 2 - 9 } & A1 & A2 & B & C & D1 & D2 & E & Total \\
\hline Lagoa & 10 & 35 & 1 & 4 & 1 & 1 & 1 & 53 \\
Centro & 8 & 30 & 4 & 4 & 1 & 1 & & 48 \\
Juazeiro & 2 & 16 & 3 & - & - & 1 & 2 & 24 \\
Total & 20 & 81 & 8 & 8 & 2 & 3 & 3 & 125 \\
\hline
\end{tabular}

Fonte: Secretaria Municipal de Saúde (adaptado).

O depósito do tipo A2 é aquele que está ao nível do solo para armazenamento doméstico de água tonel, tambor, barril, tina, depósitos de barro (filtros, moringas, potes), cisternas, caixas d'água,

\footnotetext{
${ }^{5}$ Druck et al. (2004) afirma que essa estimativa realiza uma contagem de todos os pontos dentro de uma região de influência, ponderando-os pela distância de cada um à localização de interesse, onde seus parâmetros básicos são o raio de influência que define a vizinhança do ponto a ser interpolado e uma função de estimação com propriedades de suavização do fenômeno.

${ }^{6}$ Optou-se pela correlação de Spearman em virtude das variáveis terem poucas observações $(n=14)$, além de que a variável Depósitos Positivos ter violado o teste de Shapiro-Wilk para o pressuposto da normalidade $(\mathrm{p}<$ $0,05)$. 
Carlos Vangerre de Almeida Maia Gledson Santos de Lima

Distribuição espacial de criadouros de Aedes aegypti em Alisson Daniel Silva Rocha Jaguaruana - CE - Brasil e suas correlações com Estelita Lara de Oliveira indicadores sociodemográficos

captação de água em poço/cacimba/cisterna, cujas providências a serem tomadas se centram em providenciar cobertura ou vedação; se indispensáveis, proteger/lavar, ou, em último caso descartar.

O depósito mais recorrente foi o do tipo A2; também o mais identificado no estudo de Costa et al (2016), cujos autores afirmam que esse criadouro é o mais comum em domicílios, onde, por vezes, a caixa d'água pode ser um recipiente improvisado e os moradores não os mantém devidamente vedados.

Na figura 3, à esquerda estão todos os DPs identificados para os anos de 2014 e 2015 nos bairros e, à direita, representa a tendência da ocorrência de focos, onde percebe-se maior tendência direcional no ano de 2015, pelo achatamento da elipse com direção norte-sul dos locais de ocorrência.

Figura 2 - Distribuição espacial de focos positivos e tendência de dispersão dos focos positivos

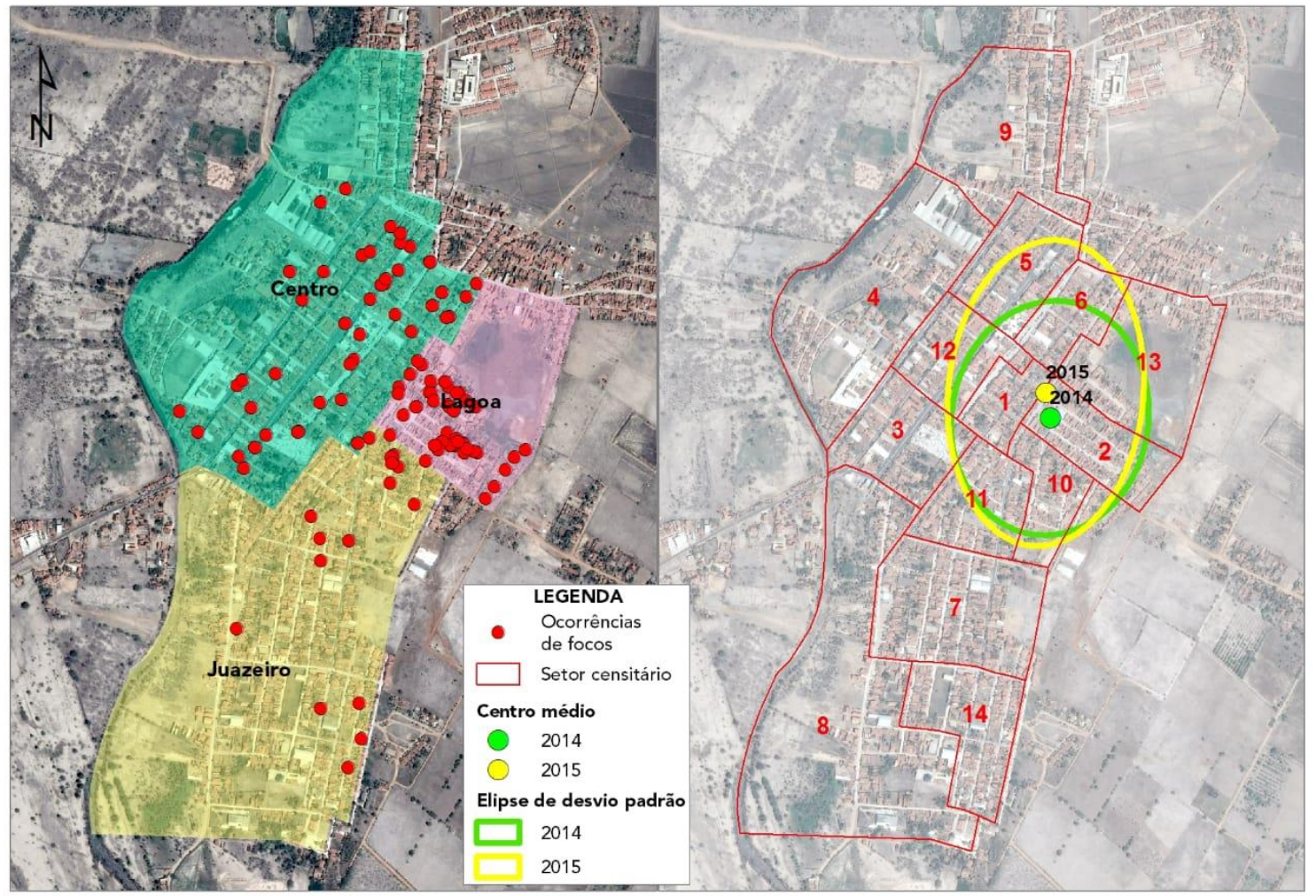

Fonte: Própria

Para Longley et al. (2013), a análise de centro médio calcula a tendência central utilizando os valores médios das coordenadas de todos os pontos e, do mesmo modo que a média, essa técnica é útil na comparação de diferentes períodos.

Já Barcellos et. al (2005) utilizaram elipses de desvios-padrão para identificar locais com potencial para transmissão de Aedes aegypti, em Porto Alegre-RS, Brasil, cuja intersecção entre as elipses de focos e acometimentos identificadas pelos autores possibilitou aventar a transmissão autóctone do vírus.

Identificou-se que para o ano de 2015 a tendência para a ocorrência de DPs foi alargada, porém mantendo contígua intersecção com a de 2014, sugerindo que, aparentemente, os esforços para a erradicação para os DPs para esse ano, não se mostraram muito eficiente, especialmente porque os centroides estão localizados no mesmo setor censitário. 
Distribuição espacial de criadouros de Aedes aegypti em Jaguaruana - CE - Brasil e suas correlações com indicadores sociodemográficos
Carlos Vangerre de Almeida Maia Gledson Santos de Lima Alisson Daniel Silva Rocha Estelita Lara de Oliveira Márcia Cristina Freitas da Silva Hihara Henrique da Silva Gabriel Douglas Santiago Barros

Uma vez especializado os focos, a Figura 4, apresenta o mapa de calor referente à densidade de Kernel, bem como apresenta um buffer de 200 metros, distância média de dispersão do culicideo ${ }^{7}$ (CARVALHO, 2011; MARTEIS et. al, 2013).

Figura 4 - Identificação de áreas de risco para DPs e possível área de dispersão do Ae. aegypti.

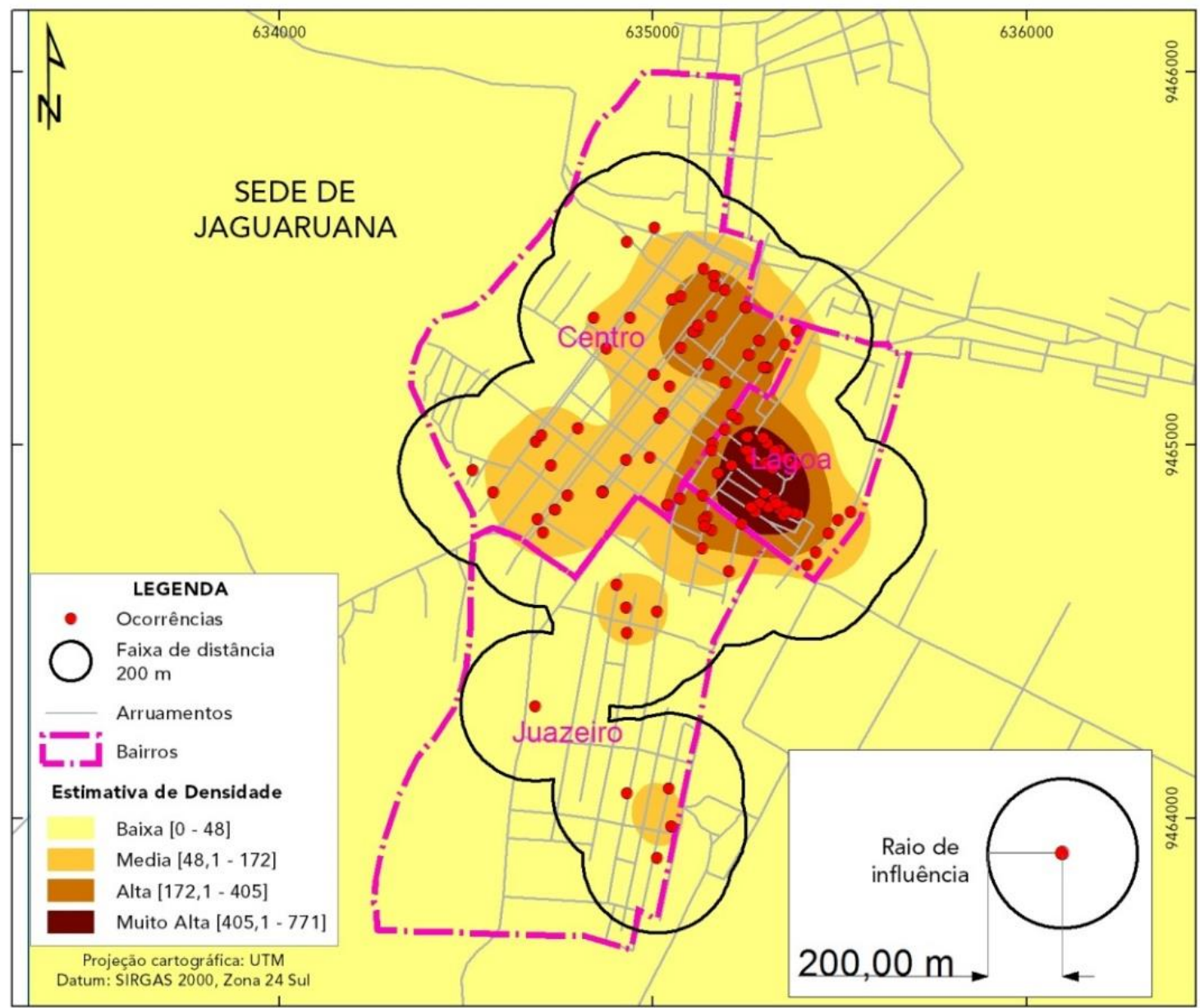

Fonte: Própria.

A área representada pelo buffer apresenta que boa parte da Sede de Jaguaruana-CE, está susceptível a dispersão do mosquito, o que pode vir a acarretar em um grave problema de saúde pública no município, enquanto que o estimador de densidade de Kernel está localizado sobre o bairro Lagoa, que se caracteriza por abrigar população economicamente hipossuficiente.

O estimador de densidade de Kernel foi uma técnica utilizada por Carvalho et al. (2017), para analisar a distribuição espacial de casos de Dengue em Rio de Janeiro - RJ, Brasil, onde foi possível constatar que as áreas com maiores densidades estão localizadas em aglomerados subnormais ou próximos a eles no município de Rio de Janeiro, sugerindo que a ocorrência da dengue pode estar relacionada à vulnerabilidade social das populações.

${ }^{7} \mathrm{~A}$ distância utilizada para o buffer foi arbitrada, uma vez que a literatura não é conclusiva quanto à dispersão do culicídeo podendo variar desde 100 a 1000 metros (SANTA CATARINA, 2018). 
Distribuição espacial de criadouros de Aedes aegypti em Jaguaruana - CE - Brasil e suas correlações com indicadores sociodemográficos
Carlos Vangerre de Almeida Maia Gledson Santos de Lima

Alisson Daniel Silva Rocha

Estelita Lara de Oliveira Márcia Cristina Freitas da Silva Hihara Henrique da Silva Gabriel Douglas Santiago Barros

Considerando o caráter antropofílico do culicídeo e a concentração de focos positivos na área estudada, é possível aventar que as condições sociodemográficas possam favorecer que o mosquito não necessite viajar grandes distâncias, nem para se alimentar, muito menos para depositar seus ovos, já que a possível presença de adensamento populacional garanta ao mosquito alimentação e depósitos suficientes para a sua perpetuação, sendo, pois, variáveis importantes para o entendimento da distribuição especial do mosquito.

Dessa feita, realizou-se correlação de Spearman, considerando o intervalo de confiança menor ou igual a 95\% $(p \leq 0,05)$ entre a presença de DPs e variáveis socioeconômicas e sanitárias nos setores censitários (renda, alfabetização, densidade demográfica e domicílios com acesso aos serviços de abastecimento de água, esgotamento sanitário e coleta de resíduos sólidos), cuja Tabela 2 apresenta os valores das variáveis que foram significativas para o teste de correlação, enquanto que a Tabela 3 apresenta a matriz de correlação de Spearman, contendo os valores de $\rho$ (rô) e da significância.

Tabela 2 - Variáveis utilizadas na Correlação de Spearman

\begin{tabular}{llccc}
\hline $\begin{array}{c}\text { Setor } \\
\text { Censitário }\end{array}$ & Bairro & $\begin{array}{c}\text { Depósitos } \\
\text { Positivos }\end{array}$ & $\begin{array}{c}\text { Densidade } \\
\text { Populacional } \\
(\text { hab/Km²) }\end{array}$ & $\begin{array}{c}\text { \% Domicílios atendidos por } \\
\text { coleta de resíduos sólidos }\end{array}$ \\
\hline 1 & Centro & 7 & 91 & 100.00 \\
2 & Lagoa & 36 & 129 & 97.00 \\
3 & Centro & 12 & 49 & 99.31 \\
4 & Centro & 3 & 35 & 98.63 \\
5 & Centro & 18 & 64 & 100.00 \\
6 & Centro & 14 & 120 & 98.54 \\
7 & Juazeiro & 2 & 55 & 100.00 \\
8 & Juazeiro & 0 & 18 & 99.55 \\
9 & Centro & 1 & 48 & 100.00 \\
10 & Juazeiro & 8 & 88 & 100.00 \\
11 & Juazeiro & 3 & 73 & 100.00 \\
12 & Centro & 2 & 60 & 100.00 \\
13 & Lagoa & 22 & 53 & 97.02 \\
14 & Juazeiro & 4 & 79 & 99.55 \\
\hline
\end{tabular}

Fonte: IBGE (2011) (adaptado) e Secretaria Municipal de Saúde de Jaguaruana - CE - Brasil

Tabela 3 - Matriz de Correlação

\begin{tabular}{|c|c|c|c|c|}
\hline & & $\begin{array}{l}\text { Depósitos } \\
\text { Positivos }\end{array}$ & $\begin{array}{l}\text { Densidade } \\
\text { Populacional }\end{array}$ & $\begin{array}{c}\% \% \\
\text { domicílios } \\
\text { com coleta } \\
\text { de lixo }\end{array}$ \\
\hline \multirow{2}{*}{ Depósitos Positivos } & $\rho$ & 1.00 & 0,58 & -0.53 \\
\hline & $p$-valor & & 0.03 & 0.05 \\
\hline \multirow[b]{2}{*}{ Densidade Populacional } & $\rho$ & 0,58 & 1.00 & -0.04 \\
\hline & $p$-valor & 0.03 & & 0.89 \\
\hline \multirow[t]{2}{*}{ \% domicílios com coleta de resíduos } & $\rho$ & -0.53 & -0.04 & 1.00 \\
\hline & $p$-valor & 0.05 & 0.89 & \\
\hline
\end{tabular}

Fonte: Própria 
De acordo com a matriz de correlação, é possível constatar que há correlação moderada inversamente proporcional entre a quantidade de DPs e o \% de domicílios atendidos com coleta de resíduos sólidos; e correlação moderada diretamente proporcional entre a quantidade de DPs e densidade demográfica.

Com isso, pode-se aventar que os serviços de saneamento básico se constituem como fator de proteção ante à ocorrência de DPs, enquanto que o maior aglomerado de pessoas se constitui como possível fator de risco para a existência de focos do culicídeo.

Feitosa et al. (2015), no intuito de traçar estratégias para o controle do mosquito, relataram a importância de se analisar indicadores sociossanitários. Já Chiaravalloti Neto et al. (1998) apontaram em seu estudo maior risco para o acometimento de dengue em áreas com maior densidade demográfica e menor renda, enquanto que Carvalho et al. (2017) afirmam que a falta de condição sanitária favorece o surgimento de potencial criadouro do culicídeo, e Sobral et al. (2017) identificaram forte correlação negativa entre coleta de lixo domiciliar $(-0,835)$ e casos de dengue na em Recife-PE.

Logo, a presença de adensamentos populacionais pode favorecer a hematofagia do mosquito, enquanto que menores coberturas do serviço de coleta de resíduos sólidos podem proporcionar a um vasto repertório de DPs para sua ovipostura, cuja sinergia pode potencializar ainda mais a perpetuação do Ae. aegypti, sendo, pois, importante analisar as variáveis que propiciam tal aspecto, visando reduzir iniquidades e subsidiar informações para tomada de decisões quanto ao combate às arboviroses.

\section{CONSIDERAÇÕES FINAIS}

Ao longo dos anos investigados, foram encontrados 125 depósitos positivos para o culicídeo nos bairros de interesse dessa pesquisa, mormente do tipo A2, que são aqueles utilizados para 0 armazenamento temporário de água para o abastecimento humano.

O Relatório da Reunião Internacional para Implementação de Alternativas para o Controle do Ae. aegypti recomendou que o controle vetorial das arboviroses deva incluir tecnologias geoespaciais, além de análises estatísticas, considerando variáveis sociossanitárias.

Seguindo essas diretrizes, observou-se que nos bairros do município de Jaguaruana que foram alvo dessa pesquisa a existência de uma possível área de risco para concentração de depósitos positivos do culucídeo, localizada no bairro Lagoa.

Também foi identificado um alongamento, na elipse do desvio padrão, representando uma tendência norte-sul para o aparecimento de DPs, cujos centroides também se encontravam nesse bairro, especificamente em um mesmo setor censitário, podendo ser uma evidência de que o combate ao mosquito necessite ser mais eficiente no município de Jaguaruana-CE.

Considerando a distribuição de DPs ao longo dos setores censitários, sobrepostos aos bairros, por meio de técnicas estatísticas, é possível aferir correlação moderada e diretamente proporcional com a densidade demográfica, bem como correlação inversamente proporcional com a coleta de resíduos sólidos.

O fato da densidade populacional se configurar como um fator de risco para a ocorrência de DPs fortalece a teoria de que maiores adensamentos populacionais asseguram a hematofagia do mosquito, ao passo que melhores índices de cobertura pode se tornar fator de proteção porque pode eliminar possíveis criadouros, minando espaços propícios para a ovipostura.

Dessa feita, conforme recomendado pelo Ministério da Saúde, a combinação de ferramentas geoespaciais com técnicas estatísticas é capaz de gerar resultados importantes para subsidiar futuras tomadas de decisão quanto ao controle do culicídeo, uma vez que possibilita compreender variáveis que tornam uma determinada região propícia à existência de depósitos positivos para o mosquito. 
Carlos Vangerre de Almeida Maia Gledson Santos de Lima

Distribuição espacial de criadouros de Aedes aegypti em

Alisson Daniel Silva Rocha Jaguaruana - CE - Brasil e suas correlações com Estelita Lara de Oliveira indicadores sociodemográficos

Márcia Cristina Freitas da Silva

Hihara Henrique da Silva

Gabriel Douglas Santiago Barros

\section{REFERÊNCIAS BIBLIOGRÁFICAS}

BARCELLOS, C.; PUSTAI, A. K.; WEBER, M. A.; BRITO, M. R. V. Identificação de locais com potencial de transmissão de dengue em Porto Alegre através de técnicas de

geoprocessamento. Revista da Sociedade Brasileira de Medicina Tropical, Uberaba, v. 38, n.

3, p. 246-250, May 2005. Disponível em:

http://www.scielo.br/scielo.php?script=sci_arttext\&pid=S0037-86822005000300008\&lng=en\&nrm=iso . Accesso em: 25 Nov. 2018. https://doi.org/10.1590/S0037-86822005000300008

BRASIL. Ministério da Saúde. Boletim Epidemiológico - Volume 47 n 15 - Relatório da Reunião Internacional para Implementação de Alternativas para o Controle de Aedes Aegypti no Brasil. Disponível em: http://portalarquivos2.saude.gov.br/images/pdf/2016/abril/05/2016-012---Relatorioreuniao-especialistas-Aedes-publica----o.pdf . Acesso em: 22/08/2011.

BRASIL. Ministério da Saúde. Boletim Epidemiológico - Volume 49 n 02 - Monitoramento dos casos de dengue, febre de chikungunya e febre pelo vírus Zika até a semana epidemiológica 52 , 2017. Disponível em: http://portalarquivos2.saude.gov.br/images/pdf/2018/janeiro/23/Boletim-2018001-Dengue.pdf. Acesso em: 22/08/2018.

BRASIL. Ministério da Saúde. Levantamento Rápido de Índices para Aedes aegypti - LIRAa para Vigilância Entomológica do Aedes aegypti no Brasil. Brasília; 2013.

CARVALHO, S.; MAGALHÃES, M. A. F. M., MEDRONHO, R. A. Análise da distribuição espacial de casos da dengue no município do Rio de Janeiro, 2011 e 2012. Revista de Saúde Publica.

2017;51:79. Disponível em: http://www.scielo.br/pdf/rsp/v51/pt_0034-8910-rsp-S1518-

87872017051006239.pdf. Acesso em: 21/08/2018.

CAVALCANTI L. P. G., BARRETO, F. K. A., OLIVEIRA, R. M. A. B., CANUTO I. F. P., LIMA, A. A. B., LIMA, J. W. O., et al. Trinta anos de dengue no Ceará: história, contribuições para ciência e desafios o cenário atual com tripla circulação de arbovírus. Journal of Health and Biological Sciences. 2018 Jan-Mar; 6(1):65-82. Disponível em:

http://periodicos.unichristus.edu.br/index.php/jhbs/article/view/1415/553. Acesso em: 23 de Nov. 2018. https://doi.org/10.12662/2317-3076jhbs.v6i1.1415.p65-82.2018

CEARÁ. Boletim Epidemiológico Febre de Chikungunya. Monitoramento dos casos de Febre de Chicungunya, 2016. Disponível em: http://www.saude.ce.gov.br/index.php/boletins\#. Acesso em: 22/08/2018.

CEARÁ. Boletim Epidemiológico. Dengue, Chicungunya e Zika. Monitoramento dos casos de dengue, chicungunya e zika até a semana epidemiológica 48, 2017. Disponível em:

http://www.saude.ce.gov.br/index.php/boletins\# . Acesso em: 22/08/2018.

CHIARAVALLOTI NETO, F., MORAES, M. S., FERNANDES, M. A. Avaliação dos resultados de atividades de incentivo à participação da comunidade no controle da dengue em um bairro periférico do Município de São José do Rio Preto, São Paulo, e da relação entre conhecimentos e práticas desta população. Caderno de Saúde Publica. 1998; 14 Supl 2:S101-9.

https://doi.org/10.1590/S0102-311X1998000600009

COSTA, A.R. da.; SANTANA, C.M.; SILVA, V.L.; PINHEIRO, J.A.F.; MARQUES, M.M.M.; FERREIRA, $P, M$. Análise do controle vetorial da dengue no sertão piauiense entre 2007 e 2011. Cadernos de saúde Coletiva, v. 24, n. 3, p.: 275-281, 2016. Disponível em:

http://www.scielo.br/pdf/cadsc/v24n3/1414-462X-cadsc-24-3-275.pdf. Acesso em: 16 Set. 2018. https://doi.org/10.1590/1414-462x201600030035

DRUCK, S.; CARVALHO, M.S.; CÂMARA, G.; MONTEIRO, A.V.M. Análise Espacial de Dados Geográficos. Brasília, EMBRAPA, 2004.

FEITOSA, F. R. S.; SOBRAL, I. S.; JESUS, E. N. Indicadores socioambientais como subsídio à prevenção e controle da Dengue. Revista Eletrônica em Gestão, Educação e Tecnologia Ambiental, v. 19, p. 351-368, 2015. Disponível em:

https://periodicos.ufsm.br/reget/article/view/18239/pdf. Acesso em: 12 Dez. 2018. 
Distribuição espacial de criadouros de Aedes aegypti em Jaguaruana - CE - Brasil e suas correlações com indicadores sociodemográficos
Carlos Vangerre de Almeida Maia Gledson Santos de Lima

Alisson Daniel Silva Rocha Estelita Lara de Oliveira Márcia Cristina Freitas da Silva Hihara Henrique da Silva Gabriel Douglas Santiago Barros

IBGE - Instituto Brasileiro de Geografia e Estatística. Base de informações do Censo Demográfico 2010: Resultados do Universo por setor censitário. Rio de Janeiro, 2011.

JOHANSEN, I. C.; CARMO, R. L. ; BUENO, M. C. D. Análise Espacial em População e Ambiente: Aplicação para o estudo da dengue em Caraguatatuba, São Paulo, em 2013. Revista Espinhaço, v. 3, p. 62-77, 2014. Disponível em: http://www.revistaespinhaco.com/index.php/journal/article/view/47. Acesso em: 04 Jan. 2019.

LONGLEY, P. A.; GOODCHILD, M. F.; MAGUIRE, D. J.; RHIND, D. W. Sistemas e Ciência da Informação Geográfica. Tradução de SCHNEIDER, A. et al. 3. ed. Porto Alegre: Bookman, 2013. $540 \mathrm{p}$.

MARTEIS, L.S.; STEFFLER, L.M.; ARAÚJO, K.C.G.M de.; SANTOS, R.C. dos. Identificação e distribuição espacial de imóveis chave de Aedes aegypti no bairro Porto Dantas, Aracaju, Sergipe, Brasil entre 2007 e 2008. Caderno de Saúde Pública, v. 29, n. 2, p368-378, 2013. Disponível em: http://www.scielo.br/pdf/csp/v29n2/23.pdf. Acesso em: 21 Dez. 2018. https://doi.org/10.1590/S0102$\underline{311 \times 2013000600023}$

SANTA CATARINA. Vigilância e controle do Aedes aegypti: Orientações Técnicas para Pessoal de Campo. Florianópolis, 2018.

SANTOS S M (Org.); SOUZA, W. V. (Org.). Introdução à estatística Espacial para a Saúde Pública. 1. ed. Brasília: Ministério da Saúde, 2008. v. 1. 120 p.

SILVA, C. E.; LIMONGI, J. E. Avaliação comparativa da eficiência de armadilhas para a captura e coleta de Aedes aegypti em condições de campo. Caderno de saúde coletiva. [online]. 2018, vol.26, n.3, pp.241-248. Aug, 2018. ISSN 1414-462X. http://dx.doi.org/10.1590/1414-462x201800030045. Disponível em: http://www.scielo.br/pdf/cadsc/v26n3/1414-462X-cadsc-1414-462X201800030045.pdf . Acesso em: 13 Abr. 2019. https://doi.org/10.1590/1414-462x201800030045

SOBRAL, M. F. F., SOBRAL, A. I. G. da P.. Casos de dengue e coleta de lixo urbano: um estudo na Cidade do Recife. Ciências e Saúde Coletiva. Disponível em: em:

http://www.cienciaesaudecoletiva.com.br/artigos/casos-de-dengue-e-coleta-de-lixo-urbano-umestudo-na-cidade-do-recife/16258?id=16258. Acesso em: 21 Out. 2018. https://doi.org/10.1590/141381232018243.10702017

TARTARUGA, I. G. P. Análise espacial da centralidade e da dispersão da riqueza gaúcha de 1970 a 2000: notas preliminares. Ensaios FEE, v. 30, p. 391-426, 2009. Disponível em:

https://www.fee.rs.gov.br/tedes/analise-espacial-da-centralidade-e-da-dispersao-da-populacao-e-dariqueza-gauchas-de-1970-a-2000-metodologias-e-notas-preliminares/. Acesso em: 20 Nov. 2018.

TONIAZZO, F.; LAVNITCKI, L. ; BAUM, C. A. A relação da evolução nos números de casos e focos da dengue com o saneamento básico no Brasil e em Santa Catarina. Revista Conversatio, v. 1, p. 497-508, 2016. Disponível em: www.celer.com.br/revistaconversatio/edicao/02/artigo21.pdf. Acesso em: 14 Nov. 2018.

ZARA, A. L. S. A.; SANTOS, S. M.; OLIVEIRA, E. S. F.; CARVALHO, R. G.; COELHO, E. G.

Estratégias de controle do Aedes aegypti: uma revisão. Epidemiologia e Serviços de Saúde. v. 25, n.2, p. 391-404, 2016. Disponível em: http://www.scielo.br/pdf/ress/v25n2/2237-9622-ress-25-0200391.pdf>. DOI: 10.5123/S1679-49742016000200017. Acesso em: 21/08/2018.

https://doi.org/10.5123/S1679-49742016000200017 